\title{
Partition into triangles revisited
}

\author{
Thinh D. NGUYeN* \\ Moscow State University \\ kosmofarmer@gmail.com \\ July 13, 2018
}

\begin{abstract}
We show that if one has ever loved reading Prasolov's books, then one can move on reading our recent article [3] and several words following to deduce that partitioning a graph into triangles is not an easy problem.
\end{abstract}

\section{Proposition}

\begin{abstract}
Classical result claims that threeAdimensional matching (3DM) is NPcomplete. This was proved in [1, Chap. 3, pp.50-52].
\end{abstract}

Proposition: $3 \mathrm{DM} \cong{ }_{p}$ PARTITION INTO TRIANGLES

Proof: At the end of this article, we capture a concise picture scanned from G\&J book. Given a 3DM instance, we construct our graph as follows. The vertex set is the same as in the hypergraph of the given instance. For each triple $\{a, b, c\}$ in the given instance, we put three edges $(a, b),(b, c),(a, c)$. By a careful scrutiny the contents in the picture, we can conclude that if one can pick any triangle in our newly constructed graph, it must also be a triple in the given 3DM instance. (Hint: Consider three cases of $a b, s s, g g$ in the picture) Q.E.D.

\section{Conclusion}

As long as we do the research on a well-known conjecture, we should recall our mathematical nature from Kvant, Prasolov-style of doing mathematics, similar to mathematics of [2] back to those beautiful days.

\section{REFERENCES}

[1] Michael R. Garey, David S. Johnson, Computers and Intractability: A Guide to the Theory of NP-Completeness

[2] Phan Dinh Dieu, Le Cong Thanh, Le Tuan Hoa, Average Polynomial Time Complexity of Some NP-Complete Problems. Theor. Comput. Sci. 46(3): 219-237 (1986)

[3] Thinh D. Nguyen, Exact Weight Perfect Matching of Bipartite Graph Problem Simplified, https://osf.io/fv9jz

* Perebor 
cral, the truth-setting and fan-out component for a variable $u_{\text {, involves }}$ "internal" elements $a_{i}[j] \in X$ and $b_{i}[j] \in Y, 1 \leqslant j \leqslant m$, which will not occur in any triples outside of this component, and "external" elements $u,[j], \vec{u},[j] \in W, 1 \leqslant j \leqslant m$, which will occur in other triples. The triples making up this component can be divided into two sets:

$$
\begin{aligned}
& \left.T_{i}=\left\{\left(\bar{u}_{i},[j], a_{i}, j\right], b_{i}[j]\right): 1 \leqslant j \leqslant m\right\} \\
& T_{i}^{f}=\left\{\left(u_{i}, j \mid, a_{j}[j+1], b_{j}(j)\right): 1 \leqslant j<m\right\} \cup\left\{\left(u_{i}[m], a_{i}[1], b,[m]\right)\right\}
\end{aligned}
$$

Since none of the internal elements $(a,|j|, b,|j|:|\leqslant j \leqslant m|$ will appear in any
PRONING NP-COMPLETENESS RESULTS

The construction is completed by means of one large "garbage collection" component $G$, involving internal elements $g_{1}[k] \in X$ and $g_{2}[k] \in Y$, $1 \leqslant k \leqslant m(n-1)$, and external elements of the form $u_{4}[j]$ and $\bar{u}_{4}[j]$ from $W$. It consists of the following set of triples:

$$
\begin{aligned}
G=\left\{\left(u, b j, g_{1}[k], g_{2}[k]\right),\left(\bar{u},[j], g_{1}[k], g_{2}[k]\right):\right. \\
1 \leqslant k \leqslant m(n-1), 1 \leqslant i \leqslant n, 1 \leqslant j \leqslant m\}
\end{aligned}
$$

literals occur in clause $c_{j}$. The set of triples making up this component is defined as follows:

$$
C_{j}=\left\{\left(u,|j|, s_{1}[j], s_{2}|j|\right): u_{i} \in c_{j}\right\} \cup\left\{\left(\bar{u}_{i}|j|, s_{1}\left[j \mid, s_{2}[j]\right): \tilde{u}_{i} \in c_{j}\right\}\right.
$$

Thus any matching $M \subseteq M$ will have to contain exactly one triple from $C_{j}$. This can only be done, however, if some $u_{i}|j|$ (or $\vec{u}_{i}[j]$ ) for a literal $u_{j} \in c_{\text {, }}$ $\left(\bar{u}_{i} \in c_{i}\right)$ does not occur in the triples in $T_{i} \cap M$, which will be the case if and only if the truth setting determined by $M^{*}$ satisfies clause $c_{j}$.

3.1 SIX BASIC NP.COMPLETE PROULEMS

From the comments made during the description of $M$, it follows immediately that $M$ cannot contain a matching unless $C$ is satisfiable. We now must show that the existence of a satisfying truth assignment for $C$ implies that $M$ contains a matching.

Let $r: U-|T, F|$ be any satisfying truth assignment for $C$. We construct a matching $M^{\prime} \subseteq M$ as follows: For each clause $c_{j} \in C$, let $z_{j} \in\left\{u_{i}, \bar{u}_{i}: 1 \leqslant i \leqslant n \mid \cap c_{j}\right.$ be a literal that is set true by, (one must exist since $t$ satisfies $c_{j}$ ). We then set 